\title{
AVALIAÇÃO DA ASSOCIAÇÃO DE ABAMECTINA E IVERMECTINA NO CONTROLE DAS HELMINTOSES GASTROINTESTINAIS EM OVINOS
}

\author{
Luiz Fernando Coelho da Cunha Filho' \\ Ricardo de Castro Martins ${ }^{2}$ \\ Patrícia Aparecida Matos de Oliveira ${ }^{3}$ \\ Priscilla Gomes Carneiro Ferreira de $\mathrm{Melo}^{4}$ \\ Amaro Mendes de Araújo ${ }^{5}$ \\ Bruno Recco ${ }^{6}$ \\ Ana Paula Mazeika ${ }^{7}$
}

CUNHA FilhO, L. F. C. da; MARTins, R. de C.; OliVEIRA, P. A. M. de; MELO, P. G. C. F. de; ARAÚJO, A. M. de; RECCO, B.; MAZEIKA, A. P. Avaliação da associação de abamectina e ivermectina no controle das helmintoses gastrointestinais em ovinos. Arq. Ciênc. Vet. Zool. UNIPAR, Umuarama, v. 18, n. 1, p. 11-15, jan./mar. 2015.

RESUMO: A helmintose gastrointestinal em ovinos é um problema crescente, devido a presença da resistência anti-helmíntica, o que dificulta sobremaneira o controle. Assim sendo, o presente trabalho teve como objetivo testar a eficácia da associação de Abamectina e Ivermectina injetável para ovinos na concentração de 3,5\% em duas propriedades norte do Paraná. As ovelhas foram randomizadas pela OPG e divididas em dois grupos, sendo $\mathrm{T}_{1}$ - Controle, $\mathrm{T}_{2}$ - associação de Abamectina e Ivermectina. Os animais foram monitorados pelo método de OPG e coprocultura no dia zero e 14, para determinar a eficácia da associação, por comparação da redução da contagem de OPG, antes e após o tratamento, e os parasitos resistentes. Os resultados encontrados foram a redução de OPG pela associação Ivermectina e Abamectina de 33,89\% e 93,74\% na primeira e segunda propriedade, respectivamente, demonstrando a eficácia do produto somente na segunda propriedade. Concluiu-se que a associação Abamectina e Ivermectina 3,5\% pode ser utilizada em propriedades na região norte do Paraná para controle da verminose, após testes de eficácia. O parasito mais incidente e, portanto o de maior resistência encontrado na análise das larvas na coprocultura foi o Haemonchus sp.

PALAVRAS-CHAVE: Avermectinas. Helmintos. Ovelhas. Resistência.

\section{EVALUATION OF THE ASSOCIATION OF ABAMECTIN AND IVERMECTIN IN THE CONTROL OF GASTROINTESTINAL HELMINTHES IN SHEEP}

\begin{abstract}
Gastrointestinal helminthes in sheep is a growing problem due to the presence of anthelmintic resistance, which greatly complicates its control. Therefore, this study aims to test the efficiency of the combination of injectable abamectin and ivermectin in sheep at a concentration of 3.5\% in two properties in the northern region of Parana. The sheep were randomly assigned by OPG and divided into two groups, where T1 - control, T2 - association of abamectin and ivermectin. The animals were monitored by the OPG method and fecal culture on day zero and fourteen to determine the effectiveness of the combination by comparing the reduction in OPG before and after treatment and the resistant parasites. Ivermectin and abamectin presented a reduction of $33.89 \%$ and $93.74 \%$ in the first and second property, respectively, demonstrating the effectiveness of the product only in the second property. It could be concluded that the association of Abamectin and Ivermectin $3.5 \%$ can be used in properties in the northern Paraná to control parasitic helminths, after efficacy tests. The most prevalent parasite and therefore, the one presenting greater resistance in the analysis of the larvae during fecal culture was Haemonchus spp.
\end{abstract}

KEYWORDS: Avermectin. Helminthes. Sheep. Resistance.

DOI: https://doi.org/10.25110/arqvet.v18i1.2015.5363

${ }^{1}$ Prof. Dr. Titular de Clinica Médica, docente do Mestrado em Saúde e Produção de Ruminantes UNOPAR-UEL/Arapongas-PR. Rodovia PR 218 - Km 01, Campus Universitário, CEP 86300-000, Arapongas, PR, Brasil. e-mail: luiz.cunha@unopar.br.

${ }^{2}$ Médico Veterinário-Arapongas-PR. Rodovia PR 218 - Km 01, Campus Universitário, CEP 86300-000,Arapongas, PR, Brasil. E-mail: ricardoc_martins@ hotmail.com

${ }^{3}$ Médica veterinária- Mestranda do curso de Saúde e Produção de ruminantes UNOPAR- UEL/Arapongas-PR. Rodovia PR 218 - Km 01, Campus Universitário, CEP 86300-000, Arapongas, PR, Brasil. E-mail: paty.vt@bol.com.br.

${ }^{4}$ Médica veterinária Mestre em Saúde e Produção de Ruminantes UNOPAR-UEL/Arapongas-PR. Rodovia PR 218 - Km 01, Campus Universitário, CEP 86300-000, Arapongas, PR, Brasil. e-mail: pirt1987@hotmail.com

${ }^{5}$ Médico veterinário- Mestrando do curso de Saúde e Produção de ruminantes UNOPAR-UEL/Arapongas-PR. Rodovia PR 218 - Km 01, Campus Universitário, CEP 86300-000, Arapongas, PR, Brasil. E-mail: amaroaraujo@hotmail.com

${ }^{6}$ Médico veterinário- Mestrando do curso de Saúde e Produção de ruminantes UNOPAR-UEL/Arapongas-PR. Rodovia PR 218 - Km 01, Campus Universitário, CEP 86300-000, Arapongas, PR, Brasil. E-mail: reccoisa@hotmail.com

${ }^{7}$ Médica veterinária- Aluna especial do curso de Saúde e Produção de ruminantes UNOPAR-UEL/Arapongas-PR. Rodovia PR 218 - Km 01, Campus Universitário, CEP 86300-000, Arapongas, PR, Brasil. E-mail: anapaulamferraz@hotmail.com 


\section{EVALUACIÓN DE LA ASOCIACIÓN DE ABAMECTINA E IVERMECTINA EN EL CONTROL DE HELMINTOS GASTROINTESTINALES EN OVINOS}

RESUMEN: Los helmintos gastrointestinales en ovejas es un problema creciente debido a la presencia de la resistencia antihelmíntica, lo que complica en gran medida el control. Así, este estudio ha tenido como objetivo evaluar la eficacia de la asociación de Abamectina e Ivermectina inyectable para ovejas a una concentración de 3,5\% en dos propiedades al norte de Paraná. Las ovejas fueron separadas por OPG y divididas en dos grupos: T1 - control, T2 - Asociación Abamectina e Ivermectina. Los animales fueron controlados por el método OPG y coprocultura en el día cero y 14, para determinar la eficacia de la combinación, por comparación de la reducción del conteo de OPG, antes y después del tratamiento, y los parásitos resistentes. Los resultados encontrados fueron la reducción de OPG por asociación de Ivermectina y Abamectina de 33,89\% y $93,74 \%$ en la primera y segunda propiedad, respectivamente, lo que demuestra la eficacia del producto sólo en la segunda propiedad. Se concluyó que la asociación Abamectina e Ivermectina 3.5\% puede ser utilizada en propiedades en la región norte de Paraná para controlar gusanos, después de los ensayos de eficacia. El parásito más común y por lo tanto el de mayor resistencia encontrado en el análisis de las larvas en el cultivo fecal fue el Haemonchus spp.

PALABRAS CLAVE: Avermectinas. Helmintos. Ovejas. Resistencia.

\section{Introdução}

Estima-se que o Brasil detenha 3,3\% do rebanho de ovinos e caprinos do mundo, sendo a população de ovinos de 16,7 milhões (IBGE, 2012). A ovinocultura é uma atividade em plena ascensão em vários estados brasileiros, caracterizada como uma alternativa econômica para pequenos e médios produtores. A produção de carne ovina em 2012 foi estimada em 85 mil toneladas, apresentando um substancial crescimento na última década (FAOSTAT, 2012).

A eficiência da produção animal está ligada a genética, fatores nutricionais, ambientais e medidas sanitárias. No aspecto sanitário, a verminose gastrintestinal é o principal problema enfrentado pela ovinocultura (SCZESNY-MORAES et al., 2010), sendo responsável por perdas econômicas decorrentes do crescimento retardado, perda de peso, diminuição da ingestão de alimentos, redução na produção e baixa fertilidade (VIEIRA, 2003).

Os anti-helmínticos são utilizados no controle das parasitoses em pequenos ruminantes e são divididos em várias classes químicas: benzimidazóis, imidotiazóis, pirimidinas, lactonas macrocíclicas (FALBO et al., 2008), porém, o uso indiscriminado de agentes químicos associado a uso contínuo e sub dosagem resulta no aparecimento de parasitos resistentes aos produtos. Medina et al. (2014) afirmam que a resistência é um fenômeno pelo qual uma droga não consegue manter a mesma eficácia frente a alguns indivíduos, devido ao efeito seletivo, a eliminação de indivíduos susceptíveis favorece a manutenção de parasitos resistentes, diminuindo o efeito do agente antiparasitário (MOLENTO, 2005).

Cunha Filho e Yamamura (1999) relataram pela primeira vez na região norte do Paraná uma alta incidência de resistência parasitária aos anti-helmínticos albendazole e ivermectina, relatando também que o parasito de maior incidência, e o mais importante a se combater é o do gênero
Haemonchus.

A diversidade de produtos de mesma base no mercado, conforme Waller (1992) induz os produtores a alternarem medicamentos, efetuando, porém, uma rotação de anti-helmínticos com o mesmo princípio ativo, sem mudar, efetivamente, as diferentes classes de anti-helmíntico. A descoberta do grupo das lactonas macrocíclicas em 1975, que compreende as avermectinas e milbemicinas, causou grande impacto no setor agropecuário, devido à possibilidade de ser utilizado em diferentes espécies animais e pela elevada eficácia contra artrópodes e nematódeos. Ivermectina, abamectina, doramectina, eprinomectina e selamectina são as avermectinas disponíveis no mercado brasileiro (COSTA, 2004).

Entre os parasitos que infectam pequenos ruminantes, estão os chamados trichostrongilídeos, pertencentes à família Trichostrongylidae, que compreende os gêneros $\mathrm{Ha}$ emonchus, sendo que a elevada prevalência associada à grande patogenicidade faz desta espécie a principal. Em seguida, em ordem de importância aparece a espécie Trichostrongylus colubriformis. Na maioria das vezes as infecções são mistas, sendo ainda comum o parasitismo dos ovinos por espécies de Cooperia spp., Oesophagostomum spp. e Strongyloides papillosus (ALVES, 2010).

O objetivo do presente estudo foi de verificar a eficácia da associação da Abamectina + Ivermectina 3,5\% no controle das helmintoses gastrointestinais de ovinos no norte do Paraná.

\section{Material e Métodos}

\section{Propriedades}

O experimento foi realizado em duas propriedades localizadas no município de Mandaguari (1) e Mauá da Serra (2), pertencentes à região norte do Paraná (Tabela 1).

Tabela 1: Médias de temperatura e pluviosidade no período durante o período de realização do experimento nos municípios de Mandaguari e Mauá da Serra, Paraná.

\begin{tabular}{cccc}
\hline Propriedades & Município & Temperatura oC & Pluviosidade mm \\
\hline 1 & Mandaguari & 24,3 & 253,3 \\
2 & Mauá da Serra & 22,3 & 236,6 \\
\hline
\end{tabular}




\section{Animais}

Foram utilizados 83 ovinos das raças Ille de France e Texel com peso médio de $45 \mathrm{~kg}$ e com idade variando entre seis e 24 meses.

Os animais foram mantidos em pastagens de grama estrela (Cynodon dactylon pers) e grama mato grosso (Paspalum notatum), sal mineral e água à vontade. Os animais não receberam nenhum método de controle parasitário 60 dias antes do início do experimento.

\section{Anti-Helmínticos}

Foi utilizada no experimento a associação de Ivermectina $2,25 \mathrm{~g}+$ Abamectina $1,25 \mathrm{~g}^{8}$, na dose $1 \mathrm{~mL} / 50 \mathrm{~kg}$ p.v. em uma única aplicação, pela via sub cutânea (SC) de administração seguindo a prescrição do fabricante.

\section{Desenho Experimental}

Os animais pertencentes a este experimento foram previamente avaliados pelo método de contagem de ovos por grama de fezes (OPG) descrito por Cringoli et al. (2004). Indivíduos com OPG igual ou superior a 200 foram selecionados para o experimento (EDDI; CORACOSTANGOLO; PEÑA, 1996), sendo randomizado, o que confere uma distribuição aleatória e com médias de OPG semelhantes.

Os ovinos foram pesados e divididos em dois grupos pelo processo de randomização que confere uma distribuição aleatória e com médias de OPG semelhantes em que $\mathrm{T}_{1}$ controle (sem tratamento) e $\mathrm{T}_{2}$ (Ivermectina + Abamectina $3,5 \%$ ). No decorrer do experimento foi efetuada uma coleta de fezes no $14^{\circ}$ dia após o tratamento, de acordo com Overend et al. (1994).

\section{Exames Coprológicos}

O material fecal foi analisado pela técnica de contagem de ovos (OPG) segundo a técnica modificada de Gordon e Whithlock (1939). Das amostras positivas analisadas foram constituídos "pool" de cada um dos grupos, e realizada a técnica de coprocultura, conforme Roberts e O’Sullivan (1950), para obtenção de larvas infectantes L3. Ambos os exames foram realizados no laboratório de Parasitologia da Universidade Norte do Paraná (UNOPAR), unidade Arapongas, Paraná.

O experimento foi realizado respeitando-se as normas do Comitê de Ética na utilização de animais em pesquisa (aprovado pelo Comitê de Ética para uso de animais: CEA/ UNOPAR $n^{\circ} 003 / 15$ ).

\section{Análise de Dados}

A partir dos valores encontrados na contagem do OPG, antes e depois do tratamento, realizou-se o teste de redução de contagem de ovos por grama de fezes (FECR Fecal Egg Count Reduction Test), utilizando-se a fórmula $\mathrm{FECR} \%=(1-\mathrm{T} 2 / \mathrm{T} 1 \times \mathrm{C} 1 / \mathrm{C} 2) \times 100$, descrita por Boersema e Pandey (1997), para determinar a eficácia dos produtos, por

${ }^{8}$ Solution LA ${ }^{\circledR}$, Intervet/Schering-Plough. comparação da redução da contagem de OPG, antes e após o tratamento, onde $\mathrm{T}_{1}$ fica sendo a média de OPG do dia zero, $\mathrm{T}_{2}$ a média do décimo quarto dia, $\mathrm{C}_{1}$ a média de OPG do dia zero do grupo controle e $\mathrm{C}_{2}$ a média do grupo controle no décimo quarto dia (TAYLOR; HUNT, 1989).

A eficácia foi considerada quando a redução de OPG foi superior a 90\% (WOOD, 1995).

\section{Resultados e Discussão}

Os resultados encontrados na propriedade 1 (Aba+iver 1 - Gráfico 1), revelaram a redução de OPG em $33,90 \%$ para a associação entre Abamectina + Ivermectina demonstrando que os anti-helmínticos testados no experimento tiveram baixa eficácia, provavelmente esta propridade possui parasitos resistentes a classe das Avermectinas, possivelmente pelo uso contínuo no controle da verminose. Na segunda propriedade (Aba+iver 2 - Gráfico 1) revelaram 93,75\% de eficiência da Abamectina + Ivermectina, demostrando a eficiência da associação. Nas duas propriedades os grupos controle não reduziram a contagem de ovos nas fezes e o percentual foi considerado zero.

Figura 1: Porcentuais de redução da OPG de ovinos submetidos ao tratamento com uma associação de Abamectina + Ivermectina em duas propriedades na região norte do Paraná

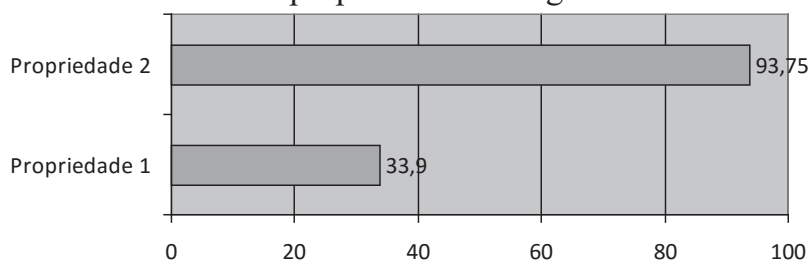

Buzzulini, Borges e Soares (2007) encontraram percentuais superiores a 95\% de redução de OPG utilizando-se da associação de Albendazole, Levamisole e Ivermectina, resultado próximo ao que foi encontrado por Lima (2010) o qual registrou índice de $100 \%$ de redução de OPG utilizando Ivermectina em ovinos.

Esses resultados diferem dos encontrados por Cardoso et al. (2009) que verificaram na região de Araçatuba, no estado de São Paulo, a resistência dos helmintos a Ivermectina, assim como Cezar et al. (2010) no Rio Grande do Sul, Ramos, Bellato e Ávila (2002) em Santa Catarina e Thomaz-Soccol et al. (2004) no Paraná os quais encontraram alta prevalência de resistência a Ivermectina e suas associações.

Molento (2004) verificou resistência lateral à Ivermectina $2,25 \%$ + Abamectina $1,25 \%$ com redução de apenas $82 \%$ no OPG, corroborando com os achados de Cunha Filho et al. (2008) que a associação closantel, albendazole e ivermectina 3,5\% tiveram baixa eficácia, reduzindo a OPG em $73 \%$ e $72,5 \%$ respectivamente.

Com relação à Ivermectina houve resistência total no presente trabalho, enquanto Piona et al. (2008) no município de Santo Antônio de Levenger-MT observaram baixo nível de redução no OPG com o uso da Ivermectina $(63,99 \%)$. Ressalta-se neste trabalho que a vermifugação anterior ao experimento realizado foi também a base da Ivermectina, albendazol e febendazol contribuindo para a ocorrência da resistência. 
Chagas (2005) acredita que a adoção de um controle supressivo de vermifugação pode acelerar o aparecimento de resistência parasitária, devido à utilização maciça de vermífugo, e sugere que a adoção do método FAMACHA (Faffa Malan Chart) seria a solução mais adequada para o controle das verminoses gastrintestinais, pois consiste em um tratamento seletivo dos animais. Já Bordin (2004) acredita que o uso de antiparasitários é um recurso necessário, porém não renovável, na medida em que a resistência avança progressivamente sobre os mais modernos grupos químicos, por isso a importância de testes de resistência e associação de produtos químicos.

De acordo com o estudo de Mexia et al. (2011), ovelhas da raça Santa Inês são menos susceptíveis à infecção parasitária frente a outras raças como Texel e Bergamácia, podendo então ser recomendada o uso desta para formação de rebanho de matrizes. Van Wyk, Malan e Bath (1997) relataram o impacto da resistência parasitária na África do Sul, Austrália e Nova Zelândia nos produtores tradicionais que diante da resistência se vêem sem alternativas e devido à baixa produtividade dos rebanhos desativam seus criatórios.

Zacharias (2004) entende que devido à resistência ser tão intensa, um plano de ação baseado apenas em vermífugos está fadado ao insucesso, pois as novas maneiras de controle privilegiam as medidas de manejo, em todos os seus aspectos, o que se refere à procura de novas formas de controle como produtos fitoterápicos e método FAMACHA. Neste sentido Silva et al. (2009) defende o uso de simbiótico a base de Saccharomyces cerevisiae para a diminuição de OPG em cordeiros confinados, enquanto Yoshihara, Minho e Yamamura (2013) acreditam num controle de nematódeos e redução na ocorrência de isolados resistentes a drogas anti-helmínticas usando tanino condensado junto com medidas de controle convencionais, visando a diminuição do uso indiscriminado de drogas.

Os resultados da coprocultura demonstraram que o gênero prevalente na população resistente foi Haemonchus sp. (95,23\%), sendo também observados os gêneros Trichostrongylus sp. e Cooperia sp., porém em menor frequência (2,97\% e 1,8\% respectivamente) corroborando com os resultados de Sczesny-Moraes et al. (2010) que encontraram 86,9\% Haemonchus $\mathrm{sp}$., 47,5\% Trichostrongylus sp. e 19,7\% Cooperia sp. resistentes a anti-helmínticos e Cunha Filho et al. (2008) os quais relataram 94,25\% de Haemonchus sp e $4,35 \%$ e 1,4\% de Trichostrongylus sp e Cooperia sp, respectivamente.

\section{Conclusão}

Concluiu-se que a associação Abamectina e Ivermectina $3,5 \%$ pode ser utilizada em propriedades na região norte do Paraná para controle da verminose, após testes de eficácia. O parasito mais incidente e, portanto o de maior resistência encontrado na análise das larvas na coprocultura foi o Haemonchus sp..

\section{Referências}

ALVES, L. R. V. Controle da verminose gastrintestinal em ovinos. Disponível em: <http://www.lanila.com.br>. Acesso em: 19 nov. 2010.
BOERSEMA, J. H.; PANDEY, V. S. Anthelmintic resistance of trichostrongylids in sheep in the highveld of Zimbabwe. Veterinary Parasitology, v. 68, p. 383-388, 1997.

BORDIN, E. L. Algumas considerações sobre a resistência de nematoides gastrointestinais de ruminantes aos antihelmínticos. In: CONGRESSO BRASILEIRO DE PARASITOLOGIA VETERINÁRIA; SIMPÓSIO LATINOAMERICANO DE RICKETTSIOSES, 13., 1., 2004. Ouro

Preto. Anais... Ouro Preto, 2004.

BUZZULINI, C.; BORGES, F. A.; SOARES, V. E. Eficácia anti-helmíntica comparativa da associação albendazole, levamisole e Ivermectina à moxidectina em ovinos.

Pesquisa Agropecuária Brasileira, v. 42, n. 6, p. 891-895, 2007.

CARDOSO, D. et al. Situação da resistência de helmintos de ovinos a anti-helmínticos na região de Araçatuba, estado de São Paulo. In: CONGRESSO BRASILEIRO DE ZOOTECNIA, 2009, Águas de Lindóia. Anais... Águas de Lindóia: FZEA/USP; ABZ, 2009.

CEZAR, A. S. et al. Multiple resistance of gastrointestinal nematodes to nine different drugs in sheep flock in southern Brazil. Veterinary Parasitology, v. 173, n. 1-2, p. 157-160, 2010.

CHAGAS, A. C. S. Prática de controle da verminose em ovinos e caprinos. Comunicado Técnico, Dez. 2005.

Disponível em: $<$ http://www.cnpc.embrapa.br $>$. Acesso em: 20 nov. 2014

COSTA, J. A. Atividade endectocida de uma inovação quimioterápica (Ivermectina + Abamectina): resultados de 12 avaliações experimentais. Revista Brasileira de Parasitologia Veterinária, Ouro Preto, v.13, suplemento 1, 2004.

CRINGOLI, G. et al. The influence of flotation solution, sample dilution and the choice of McMaster slide area (volume) on the reabilifty of the McMaster technique in estimating the fecal egg counts of gastrointestinal strongyles and Dicrocoelium dendriticum in sheep. Veterinary Parasitology, v. 123, p. 121-131, 2004.

CUNHA FILHO, L. F.; YAMAMURA, M. H. Resistência a anti-helmínticos em ovinos da região de Tamarana. Paraná, Brasil. Ciência Biológica da Saúde, Londrina, v. 1, n. 1, p. 31-39, 1999.

CUNHA FILHO, L. F. et al. Eficácia da associação closantel albendazol e ivermectina 3,5 no controle da helmintose de ovinos da Região Norte do Estado do Paraná. Ciência Biológica da Saúde, Londrina, v. 10, n. 2, p. 23-28, 2008.

EDDI, C.; CARACOSTANGOLO, J.; PEÑA, M. The prevalence of anthelmintic resistence in nematode parasites of sheep in Southern Latin America: Argentina. Veterinary Parasitology, v. 62, p. 189-197, 1996. 
FALBO, M. K. et al. Atividade anti-helmíntica do fruto de Melia azedarach em cordeiros naturalmente infectados com nematódeos gastrintestinais. Semina: Ciências Agrárias, v. 29 , n. 4, p. 881-886, 2008.

FAOSTAT - Organização das Nações Unidas para a Alimentação e a Agricultura. 2012. Disponível em: $<$ https:// faostat.fao.org.br>. Acesso em: 27 mar. 2015.

GORDON, H. M.; WHITLOCK, H. V. A. new technique for counting nematode eggs in sheep faeces. Journal Council Scientific Industrial Research, v. 12, p. 50-52, 1939.

IAPAR - Instituto Agronômico do Paraná. Disponível em: $<$ https:// www.iapar.br>. Acesso em: 19 nov. 2014.

IBGE - Instituto Brasileiro de Geografia e Estatística. Disponível em: <ftp://ftp.ibge.gov.br/Producao_Pecuaria/ Producao_da_Pecuaria_Municipal/2012/ppm2012.pdf $>$. Acesso em: 27 mar. 2015.

LIMA, W. C. et al. Nematóides resistentes a alguns anti-helmínticos em rebanhos caprinos no Cariri Paraibano. Pesquisa Veterinária Brasileira, v. 30, n. 12, p. 1003-1009, 2010.

MEDINA, P. et al. Resistencia antihelmíntica en ovinos: una revisión de informes del sureste de México y alternativas disponibles para el control de nemátodos gastrointestinales. Pastos y Forrajes, v. 37, n. 3, p. 257-263, 2014.

MEXIA, A. A. et al. Susceptibilidade a nematoides em ovelhas Santa Inês, Bergamácia e Texel no Noroeste do Paraná. Londrina. Semina: Ciências Agrárias, v. 32, suplemento 1, p.1921-1928, 2011.

MOLENTO, B. M. Resistência de helmintos em ovinos e caprinos. In: CONGRESSO BRASILEIRO DE PARASITOLOGIA VETERINÁRIA; SIMPÓSIO LATINOAMERICANO DE RICKETISIOSES, 13., 1., 2004, Ouro Preto. Anais... Ouro Preto, 2004.

Resistência parasitária em helmintos de equídeos e propostas de manejo. Ciência Rural, v. 35, n. 6, p. 1469$1477,2005$.

OVEREND, D. J. et al . Antihelmintic resistence in Australian sheep nematode populations. Australian Veterinary Journal, v. 71, p. 117, 1994.

PIONA, M. N. M. et al. Resistência anti-helmíntica em ovelhas da raça Santa Inês criadas a pasto no município de Santo Antônio de Leverger - MT. João Pessoa. UFPB/ ABZ 2008.

RAMOS, C. I. et al. Resistência de parasitos gastrintestinais de ovinos a alguns anti-helmínticos no Estado de Santa Catarina, Brasil. Ciência Rural, v. 32, n. 3, p. 473-477, 2002. egg counts and larval cultures for strongyles infesting the gastrointestinal tract of cattle. Australian Agriculture Research, v. 1, p. 99-102, 1950.

SCZESNY-MORAES, E. A. et al. Resistencia antihelmíntica de nematoides gastrintestinais em ovinos, Mato Grosso do Sul. Pesquisa Veterinária Brasileira, v. 30, n. 3, p. 229-236, 2010.

SILVA, K. C. F. et al. Efeito de um simbiótico sobre o ganho de peso e número de ovos por grama de fezes de cordeiros confinados. Semina: Ciências Agrárias, v. 30, n. 4, p. 953-962, 2009.

TAYLOR, M. A.; HUNT, K. R. Anthelmintic drug resistance in the UK. Veterinary Research, v. 125, p. 143$147,1989$.

THOMAZ-SOCCOL, V. et al. Resistance of gastrointestinal nematodes to anthelmintics in sheep (Ovis aries). Curitiba. Brazilian Archives of Biology and Technology, v. 47, n. 1, p. 41-47, 2004.

VAN WYK, J. A.; MALAN, F. S.; BATH, G. F. Rampant anthelmintic resistance in sheep in South Africa - what are the options? Workshop Managing anthelmintic resistance in endoparasites. In: INTERNATIONAL CONFERENCE OF THE WORLD ASSOCIATION FOR THE ADVANCEMENT OF VETERINARY PARASITOLOGY. 16., 1997, Sun City. Anais... Sun City, África do Sul, 1997. p. 51-63.

VIEIRA, L. S. Alternativas de controle de verminose gastrintestinal dos pequenos ruminantes. Brasília: EMBRAPA, n. 29, p. 1-10, 2003.

WALLER, P. J. Resistência dos nematóides aos antihelmínticos na Austrália. A Hora Veterinária, v. 12, n. 69, p. 24-26, 1992.

WOOD, I. B. et al. World Association for the Advancement of Veterinary Parasitology (W.A.A.V. P.) second edition of guidelines for evaluating the efficacy of anthelmintics in ruminants (bovine,ovine, caprine). Veterinary

Parasitology, v. 58, p. 181-213, 1995.

YOSHIHARA, E.; MINHO, A. P.; YAMAMURA, M H. Efeito anti-helmíntico de taninos condensados em nematódeos gastrintestinais de ovinos (Ovis aries). Semina: Ciências Agrárias, v. 34, n. 6, p. 3935-3950, 2013.

ZACHARIAS, F. Parasitismo em pequenos ruminantes: novos conceitos. Bahia Agrícola, v. 6, n. 3, nov. 2004.

Recebido em: 19.03.2015 Aceito em: 31.03.2015 